\title{
Uma Fisiologia da Paisagem II: Percepção e Movimento
}

\author{
José Roberto Pellini*
}

PELLINI, J.R. Uma Fisiologia da Paisagem II: Percepção e Movimento. Revista do Museu de Arqueologia e Etnologia, São Paulo, 18: 3-18, 2008.

Resumo: Pensando que percepção é uma função do movimento, podemos dizer que a maneira com a qual os indivíduos percebem seu entorno depende de como elas se movem no meio. Se através do movimento os indivíduos alteram sua percepção do espaço, a dimensão espacial em arqueologia pode ser conceituada a partir dos perfis de mobilidade dos indivíduos. Hoje em dia as metodologias de análise em GIS possibilitam a reconstrução dos padrões de mobilidade humana utilizando as mais recentes pesquisas em fisiologia do movimento e as chamadas análises de visibilidade. Isto possibilita o desenvolvimento de abordagens que privilegiam aspectos cognitivos relacionados à percepção e movimento e, assim, a criação de ferramentas no GIS que vão além das noções cartesianas de espaço.

Palavras-chave: GIS - Percepção - Movimento - Paisagem.

\section{Introdução: um ajuste no olhar}

Nos últimos anos tem crescido consideravelmente o uso dos chamados Sistemas de Informação Geográfica (GIS) como ferramenta de análise em arqueologia. Mas a despeito do seu uso cada vez mais freqüente, muitos pesquisadores ainda olham com desconfiança os resultados das análises.

A principal crítica vem daqueles que consideram o GIS uma ferramenta de análise determinista. O problema a nosso ver não está no GIS, mas nos aportes teóricos que guiam as análises, principalmente no que se refere à definição de espaço. Como apontam Gillings e

${ }^{*}$ ) Instituto de Pré-História e Antropologia (IGPA) Universidade Católica de Goiás.

$\left({ }^{*}\right)$ Griphus Consultoria em Arqueologia.

<jrpellini@yahoo.com.br>
Goodrick (1996), a maioria dos estudos que aplicam o GIS em arqueologia utiliza uma noção de espaço que é estritamente cartesiana, onde espaço é visto como universal, mensurável e externo à atividade cultural. Neste sentido o espaço é tratado como elemento neutro e sem nenhum valor cultural, uma dimensão desumanizada caracterizada por linhas, pontos e planos. Esta perspectiva cartesiana tem limitado o potencial de análise do GIS já que esta geografia estéril falha em representar alguns aspectos das sociedades ou mesmo falha em representar processos geográficos mais complexos (Miller e Wentz 2003).

Em arqueologia, nas concepções mais tradicionais, principalmente aquelas ligadas à escola processualista, o espaço existe independentemente da presença do observador. $\mathrm{O}$ meio desta forma é uma área neutra dentro da qual estruturas ecológicas e processos podem ser identificados. Neste sentido, o espaço neolítico é 
idêntico ao espaço bizantino, o espaço romano é igual ao espaço guarani, podendo ser descrito como pontos, planos e vetores. Esta concepção de um espaço que é físico, estático e universal tem dominado a grande maioria dos estudos que aplicam o GIS em arqueologia.

Mas se pensarmos que o espaço precisa ser entendido nos termos relevantes para os indivíduos que o cercam, não apenas nos termos físicos, filosóficos e matemáticos como planos, pontos e vetores, mas nos termos que mais adequadamente descrevem as experiências no mundo, na forma de superfícies, lugares e caminhos, precisamos de um novo grupo de termos para descrever a geometria de um espaço que não é isotrópico, abstrato e universal, mas um espaço no qual humanos e outros animais se movem, percebem e atuam. Enquanto o meio físico e sua estrutura invariante é real, os valores e significados do meio experimentado só podem ser compreendidos por referência aos indivíduos que percebem e reconhecem aqueles significados e valores. Desta maneira, falamos de superfícies e não de planos, falamos de locais e não de pontos, falamos de caminhos e não de linhas.

O desenvolvimento de abordagens que privilegiem aspectos cognitivos principalmente aqueles relacionados à percepção e movimento são uma tentativa de utilizar as ferramentas do GIS além das noções cartesianas de espaço e começar a buscar formas alternativas de análise.

\section{Movimento e percepção: as chaves de um novo GIS}

Bender (2001) define paisagem como um espaço que é mediado pela percepção subjetiva e sensorial dos indivíduos, sendo um elemento construído e apropriado socialmente. Nesta visão o código espacial é apreendido a partir de contingências sensoriais e motoras a partir da relação entre a movimentação do observador e suas conseqüências sensoriais. Segundo Gibson (1979), é através do aparato sensorial e do movimento que os indivíduos obtêm informações e percebem o espaço a sua volta. Para o autor movimento confere uma perspectiva múltipla e não estática ao processo de percepção, possibilitando que os indivíduos captem o mundo de diversos ângulos.

Em um primeiro momento a paisagem é formada por um fazer e apreendida por um olhar. Em nenhuma outra situação este fazer e este olhar são mais evidentes que no caminhar. $\mathrm{O}$ caminhante experimenta a profundidade material da proximidade do meio através da visão binocular e interage através do efeito de movimento criado pelo contraste dos objetos mais próximos e aqueles mais distantes. $\mathrm{O}$ mundo material próximo, sentido, tocado, cheirado, é apreendido pelo aparato sensorial do caminhante levando-o a experimentar a paisagem como um domínio topológico de lugares contínuos.

Como salientou Bourdieu (1977), caminhar envolve gestos e posturas particulares, que são um reflexo da práxis cultural de um individuo. $\mathrm{O}$ modo de caminhar não expressa meramente pensamentos e sentimentos que têm sido apropriados a partir de uma educação cultural, ele é em si uma maneira de pensar e sentir através da qual na prática pedestre as formas culturais são continuamente geradas. Entre os Batek, por exemplo, caminhar compreende um grupo de gestos corporais que incluem observar, monitorar, escutar, relembrar, tocar, cheirar, e é através destas performances que durante as caminhadas seu conhecimento do mundo é formado (Tuck Po 2008). Ao mesmo tempo em que caminham os Batek olham o topo das árvores, os galhos caídos, o entorno, procuram sinais no meio que remetem a lembranças de caminhos e experiências que ajudam a formatar sua visão de mundo. Uma caminhada é assim uma experiência de integração com o meio, com a paisagem, com a topografia. A prática do caminhar é em si um meio de educar e aprender, um meio de conhecer, interagir e socializar. Para Legat (2008), caminhar é uma experiência que liga narrativas que servem para a aquisição de conhecimento pessoal, validando histórias do passado no presente e estabelecendo uma ligação entre os espaços, as histórias e todos os seres que se utilizam de um determinado local. Enquanto caminhamos evocamos imagens da memória, viajamos não apenas no caminho imediato, mas a lugares distantes, caminhamos de volta ao 
passado e a lugares que nos remetem a cenas distantes, a cheiros, a sons e lembranças, medos e sensações não visuais que são estimuladas pelos signos de nossa jornada. Caminhar é um exercício de observação. Caminhar é um exercício de memória e de lembranças, onde você pensa como vê e vê como pensa.

Mas movimento não é uma estrutura aleatória, pois o meio ambiente concreto, real, contém formas naturais de deslocamento (Schlee 1992). A posição do relevo, a vegetação, os cursos dos rios, os tipos diferentes de solo, a posição de lagos e das calhas de drenagem, assim como outros elementos naturais dificultam ou facilitam o movimento natural de homens e animais. Esta pré-determinação natural do terreno foi chamada por Mackie (2001) de Direcionalidade Inerente do Meio. Esta Direcionalidade Inerente pode ser definida como aquelas características meio ambientais que por sua especificidade atraem ou repelem o deslocamento. Se pensarmos em uma planície, por exemplo, como o ambiente em geral possui as mesmas características e condições, o acesso a um grupo de pontos a partir de um ponto inicial, será semelhante, ou seja, neste caso o ambiente não condiciona o deslocamento; sendo assim, podemos dizer que a planície em questão apresenta baixa direcionalidade. Em contrapartida, em uma área montanhosa os vales tendem a atrair o movimento, sendo assim, a direcionalidade passa a afetar a mobilidade inevitavelmente. Direcionalidade é uma função da tecnologia e do sistema de transporte. Isto porque tecnologia e transporte podem alterar a permeabilidade do meio permitindo o acesso a lugares de difícil acesso. Fatores sociais, econômicos e culturais também geram direcionalidade na medida em que atraem movimento, como no caso, por exemplo, da exploração de minas de ouro, ou da existência de centros cerimoniais, ou repelem o movimento, como no caso, por exemplo, de áreas de tabu. A direcionalidade do movimento ocorre dentro de contextos espaciais e temporais definidos sendo mitigados e percebidos a partir de comportamentos culturais (Mackie 2001). Devemos considerar que todo terreno possui uma malha possivel de movimento, o que torna o terreno permeável, permitindo a humanização do entorno. O deslocamento dá início a um movimento que é, antes de mais nada, experiência do espaço que tem lugar em uma extensão espacial e temporal concreta. Neste sentido, movimento se torna um mecanismo para a percepção do meio.

Analisando a locomoção de certo indivíduo, podemos determinar de antemão que somente uma viagem é possível em um momento específico de tempo. Neste momento o indivíduo é confrontado com um grande número de possíveis destinos, direções e distâncias, mas evidentemente apenas uma escolha pode ser feita. Mesmo que seu movimento não possa ser determinado com precisão, sabemos que o destino escolhido é influenciado por uma relação entre informação e distância (Morrill e Pitts 1967). A informação consiste no conhecimento que um indivíduo tem do seu mundo, e seu campo de informação é a distribuição espacial deste conhecimento. Mas como sugeriu Hägerstrand (1962), a probabilidade de um indivíduo se mover de um local para o outro precisa ser computada também como uma função das características do indivíduo (idade, raça, sexo, vida urbana ou rural etc.), das características da área estudada (formas de relevo, paisagens, formações urbanas, contexto sócio-econômico), das características dos possíveis destinos bem como das diferenças entre eles.

Pensando que percepção é uma função do movimento, como defendem Ingold (1986, 2000), Tilley (1994), Gibson (1979) e Mackie (2001), podemos dizer que a maneira com a qual os indivíduos percebem seu entorno depende de como elas se movem no meio. Se através do movimento os indivíduos alteram sua percepção do meio, a dimensão espacial em arqueologia pode ser conceituada a partir dos perfis de mobilidade dos indivíduos. Movimento cria um senso de espacialidade que não ocorre em um espaço cartesiano vazio. $O$ meio existe somente em relação aos observadores. Estes observadores são móveis, sendo assim, sua percepção e seu conhecimento sobre o meio são experimentados através do movimento. Para Mackie (2001: 2), por razões sociais ou meio ambientais, alguns vetores de movimento são 
mais favoráveis do que outros, sendo assim rotas individuais se tornam redes de mobilidade coletiva que permitem interação e auxiliam na delimitação do conhecimento público. Estas rotas são ao mesmo tempo estrutura e estruturadas pelo meio ambiente construído. Através do caminhar nos tornamos parte da paisagem, não apenas um habitante, mas um caminhante (Moles 2008).

Mas se movimento e ação estabelecem as bases da percepção, para saber como o homem percebe o meio precisamos primeiramente saber como ele se move na paisagem.

\section{Como caminhamos: fisiologia do movimento}

A fisiologia do movimento tem sido muito estudada nos últimos anos. Tanto a medicina quanto a física têm analisado exaustivamente os efeitos do deslocamento humano a pé, principalmente quando se consideram caminhamentos que envolvem o transporte de carga extra (Legg e Mahanty 2004). Em particular, o efeito das cargas sobre o consumo de energia tem recebido bastante atenção. Legg (1985), por exemplo, calculou o efeito energético de diferentes tipos de carga sobre o corpo humano. Já Pandolf et al. (1979) determinaram quais os coeficientes de friç̦ão envolvidos no transcurso de diferentes tipos de terreno. Santee et al. (2003) têm trabalhado com o custo metabólico envolvido no movimento humano de acordo com os gradientes de inclinação do terreno.

Estudos fisiológicos têm mostrado que o custo metabólico de locomoção, tipicamente medido como a taxa de massa específica de consumo de oxigênio $\left(\mathrm{ml} \mathrm{O}_{2} \mathrm{Kg}^{1} \mathrm{~S}^{-1}\right)$, deriva primariamente da força muscular gerada para possibilitar o movimento e suportar o peso do corpo. Pontzer (2007) propõe um modelo em que parte do custo metabólico associado com o deslocamento em humanos está relacionada à força muscular produzida para acelerar o centro de massa corporal. Neste modelo a taxa de produção de força, calculada como força média por passo $\mathrm{x}$ freqüência de passos, sugere que o volume de músculos ativados por passos é que determina o custo de locomoção. Sendo assim podemos dizer que é o volume de músculos ativados por ciclos de passos que determina o custo de deslocamento.

Mas para Minetti et al. (1994), os fatores mecânicos que determinam o custo de deslocamento são mais complexos. Para o autor é a inter-relação entre o trabalho muscular positivo e negativo necessário nos diferentes gradientes de declividade o principal fator responsável pelo gasto metabólico associado ao deslocamento a pé. Isto porque em ambientes planos, o centro de massa corpórea associado à locomoção é igualmente balanceado entre o trabalho positivo (levantar) e negativo (abaixar). $\mathrm{O}$ trabalho positivo tende a dominar o custo metabólico nos deslocamentos morro acima enquanto o trabalho negativo domina os movimentos morro baixo, sendo que o trabalho muscular positivo tem um custo metabólico cinco vezes maior que o trabalho negativo (Abbot et al. 1952).

Durante caminhadas o gradiente de declividade é o principal responsável pelo custo metabólico ao passo que em corridas o custo é majoritariamente determinado pelo trabalho mecânico. Em movimentos mais lentos como em caminhadas o balanço entre os membros se assemelha a um pêndulo invertido. Tal movimento resulta em uma troca entre energia potencial gravitacional e a energia cinética de avanço do centro de massa. Já em movimentos mais acelerados como em corridas há uma troca entre energia elástica e a energia cinética, ou seja, no caminhamento o custo de gerar força muscular para suportar o peso do corpo tem menos importância na determinação do consumo metabólico do que em corridas. Desta maneira, o custo metabólico associado ao movimento humano varia não apenas em relação à velocidade mas principalmente em relação à inclinação do terreno sendo que o menor custo metabólico se encontra em deslocamentos em áreas planas a uma velocidade de $1,3 \mathrm{~m} / \mathrm{s}$. Já o custo metabólico associado às corridas é independente da velocidade.

Segundo Minetti et al. (2002), o custo metabólico associado à caminhada e às corridas em declividades positivas acima de $15 \%$ são proporcionais ao aumento da declividade. Em declividades negativas superiores a $-15 \%$ a relação entre custo metabólico e 
declividade se tornam lineares. No caso das caminhadas o gradiente de inclinação que minimiza o custo metabólico se dá entre 25 e $28 \%$, tantos em movimentos em declividade positiva quanto em declividades negativas. Segundo Minetti et al. (1994), o custo de se caminhar $1 \mathrm{~km}$ é minimizado em gradientes de $-10 \%$ aumentando proporcionalmente conforme aumenta o gradiente tanto em declividades negativas quanto em declividades positivas. O custo metabólico por unidade caminhada é dependente do gradiente de inclinação, mas não da velocidade exceto para declividades entre 10 e $15 \%$ onde o custo metabólico decai em altas velocidades. Segundo Tobler (1993), a relação entre velocidade e declividade pode ser expressa segundo a função:

$\mathrm{V}=6 \exp (-3,5(\mathrm{~S}+0,05))$

onde (V) representa a velocidade em $\mathrm{km} / \mathrm{h}$, (S) é a declividade do terreno e (exp) é a base natural do logaritmo. O grande problema com a função de Tobler é que ela não leva em conta nem as características do indivíduo na caminhada nem os diferentes tipos de terreno. Pandolf et al. (1979) sugeriram uma função onde o gasto metabólico pode ser definido por:

\section{$\mathrm{M}=1,5+2(\mathrm{~W}+\mathrm{L})(\mathrm{W} \mathrm{L})^{2}+\mathrm{N}(\mathrm{W}+\mathrm{L})\left(1,5 \mathrm{~V}^{2}+0,35 \mathrm{VG}\right)$}

onde (M) é a taxa metabólica medida em watts, (W) é o peso corporal do indivíduo, (L) é o peso da carga extra, (V) é a velocidade de deslocamento, $(\mathrm{G})$ é a declividade do terreno e $(\mathrm{N})$ o fator de terreno. ${ }^{1}$ Seguindo o modelo de Pandolf et al. (1979), Morin et al. (2005) propuseram uma fórmula expressa por:

$\mathrm{VO} 2=2,559+0,107\left(\mathrm{I}^{2}\right)+0,335\left(\mathrm{~S}^{2}\right)+0,182(\mathrm{~L})$

(1) Para cada tipo de terreno Pandolf et al. (1977) definiram um coeficiente de deslocamento baseado no fato de que quanto maior a penetração no solo maior o gasto de energia. Sendo assim, o fator $\mathrm{N}$ de deslocamento varia entre o coeficiente 4,1 para terrenos de neve fina até 1,0 para lajes rochosas lisas. onde (I) é a inclinação do terreno, (S) é a velocidade de deslocamento e (L) é a carga extra. A vantagem da fórmula de Morin et al. (2005) é que, diferentemente da fórmula de Pandolf et al. (1979), ela é medida em volume de oxigênio consumido, uma unidade que representa melhor o consumo metabólico do que a unidade de medida watts.

Tanto a função de Pandolf et al. (1979) quanto a função de Morin et al. (2005) tentam recriar a partir de modelos fisiológicos os padrões de deslocamento humano.

\section{São Desidério: caminhos e caminhantes}

A cidade de São Desidério na Bahia se localiza em uma das regiões cársticas mais importantes do Brasil. A área é toda dominada por grutas e lagoas. Aí se localiza o maior lago subterrâneo do mundo bem como os maiores salões já registrados no Brasil. Durante os trabalhos associados ao Projeto de Resgate Arqueológico, Histórico e Cultural da Área Direta e Indiretamente Afetada pala Implantação da Rodovia BR 135 (Pellini e Telles 2008) executado pela Griphus Consultoria sob a coordenação geral dos arqueólogos Dr. José Roberto Pellini, Márcio Antônio Telles e Me Jonas Israel Souza, no levantamento e resgate na área do empreendimento que é de responsabilidade do DNIT, foram identificados 32 sítios arqueológicos na área do cárste de São Desidério, sendo quatro sítios a céu aberto e 28 sítios em abrigo sob rocha e grutas (Mapas 01 e 02; Figs. 1 e 2).

Partindo do pressuposto de que os abrigos rupestres funcionam como um foco de atração do movimento, iremos analisar em que medida os abrigos com pintura modificavam e condicionavam a direcionalidade inerente do movimento, através da comparação das rotas de direcionalidade inerente com as rotas de direcionalidade cultural. A hipótese é que fatores culturais tendem a alterar a Direcionalidade Inerente do ambiente.

O ponto de partida para isso é determinar quais as rotas naturais existentes dentro da área de estudo. Para isso utilizamos um Modelo de Elevação Digital (DEM) da área de estudo com 

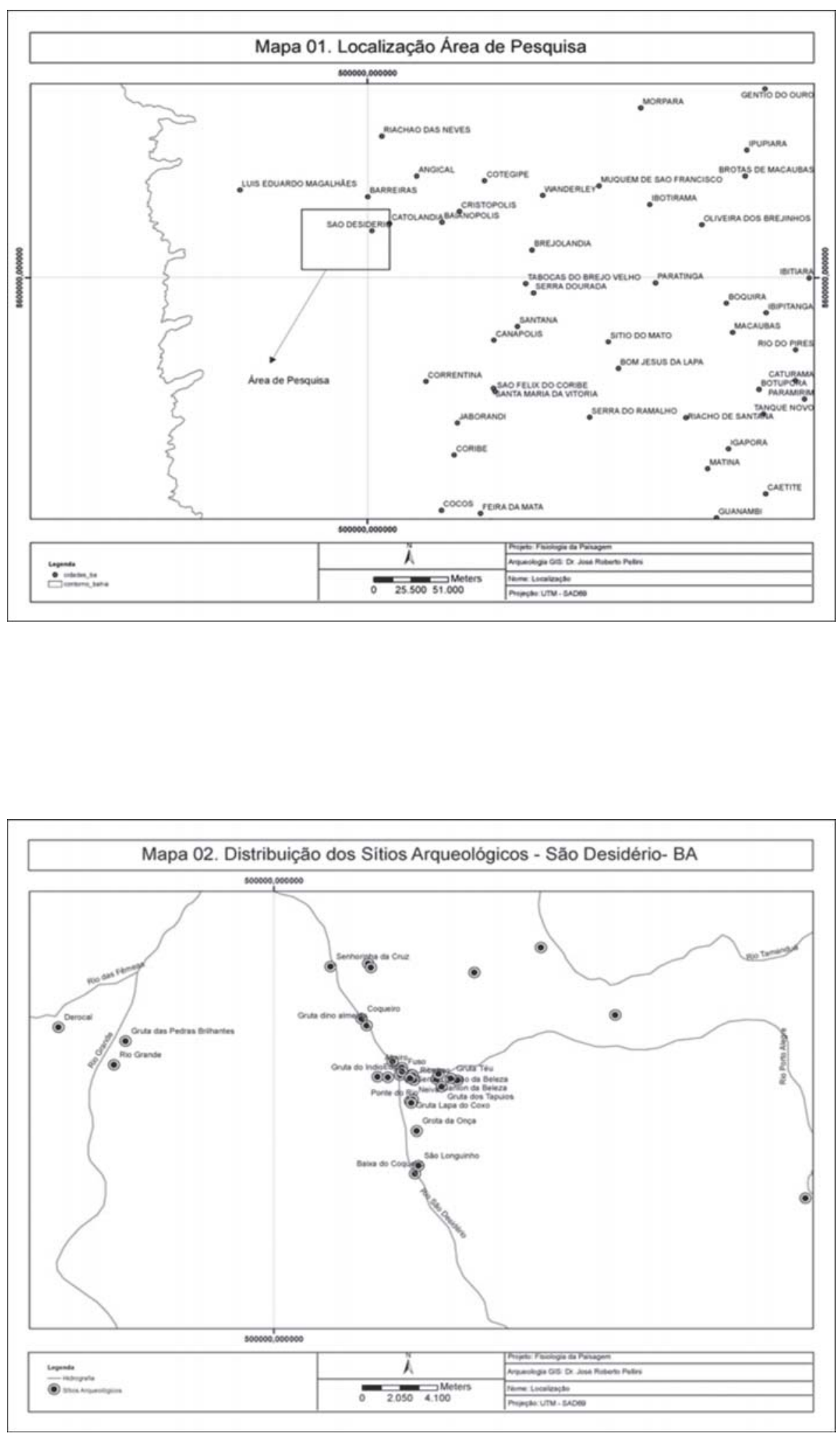

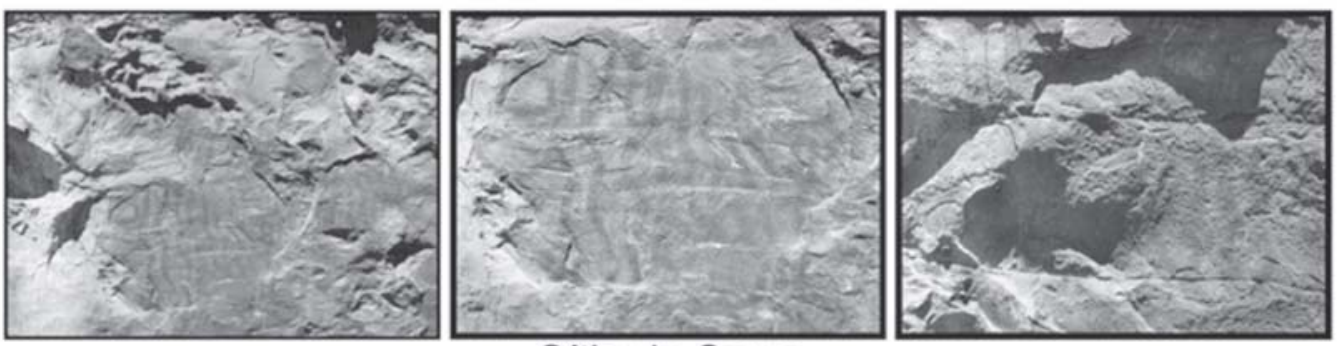

Sítio da Serra
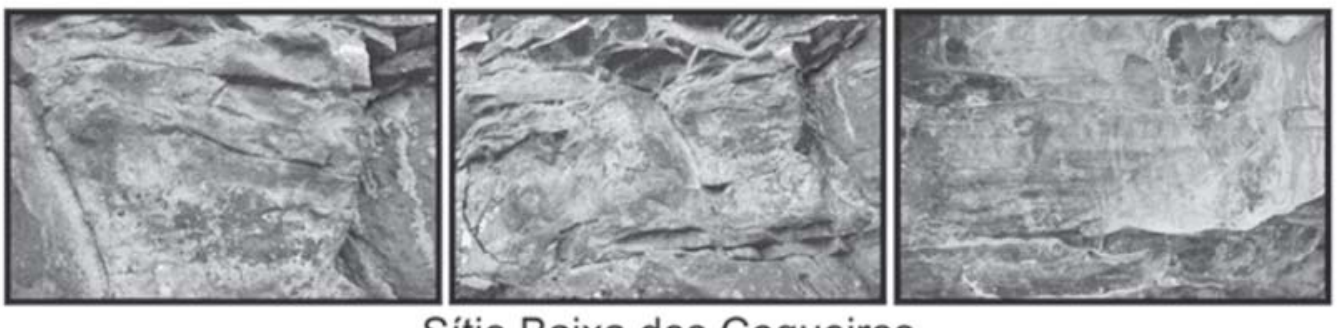

Sítio Baixa dos Coqueiros
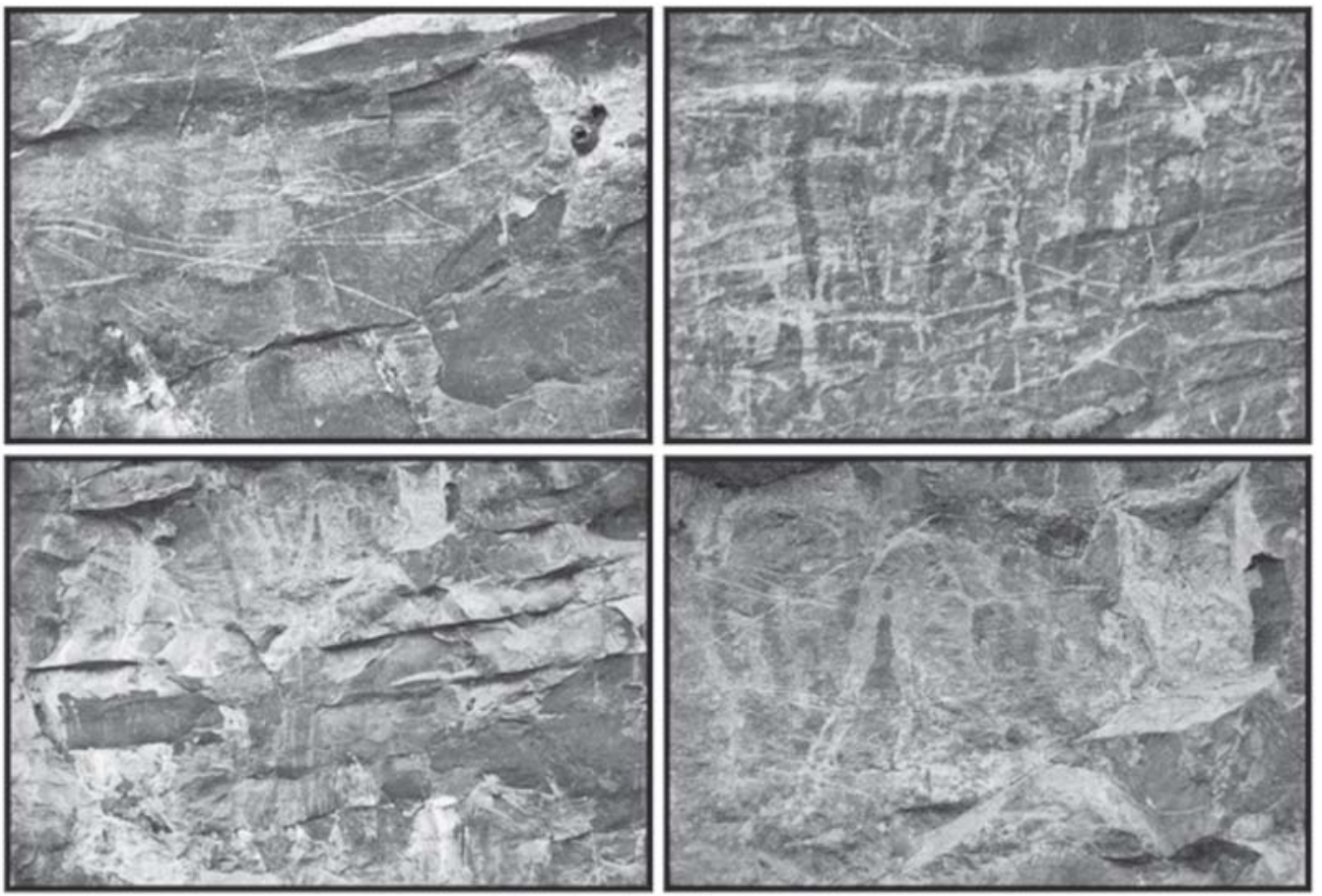

Gruta da Derocal

Fig. 1 
Uma Fisiologia da Paisagem II: Percepção e Movimento.

Revista do Museu de Arqueologia e Etnologia, São Paulo, 18: 3-18, 2008.
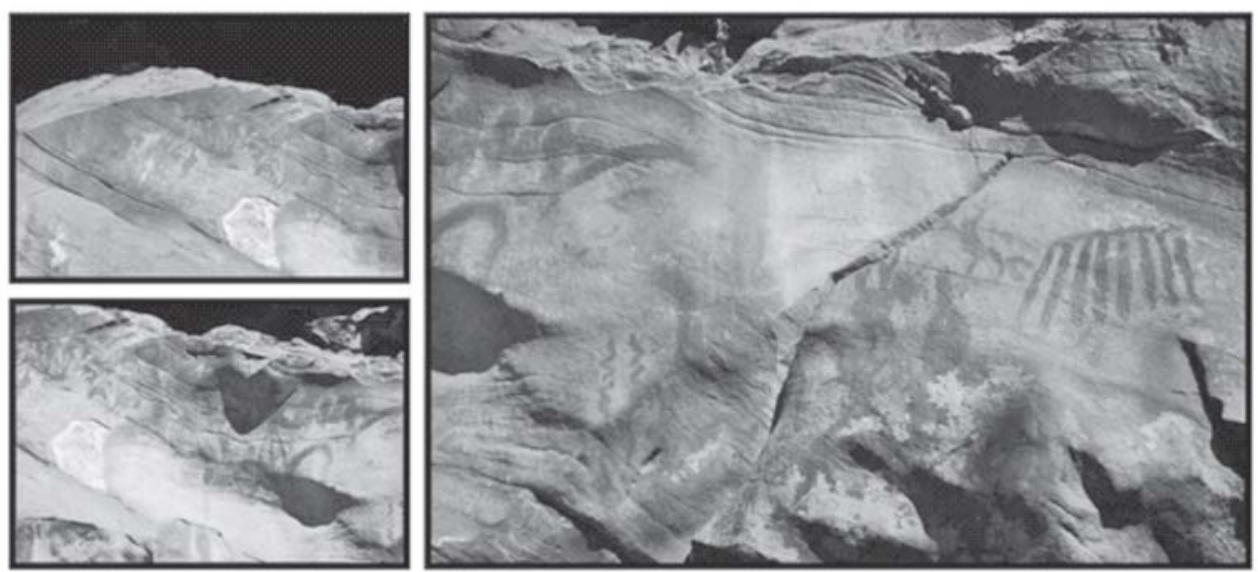

Pedras Brilhantes
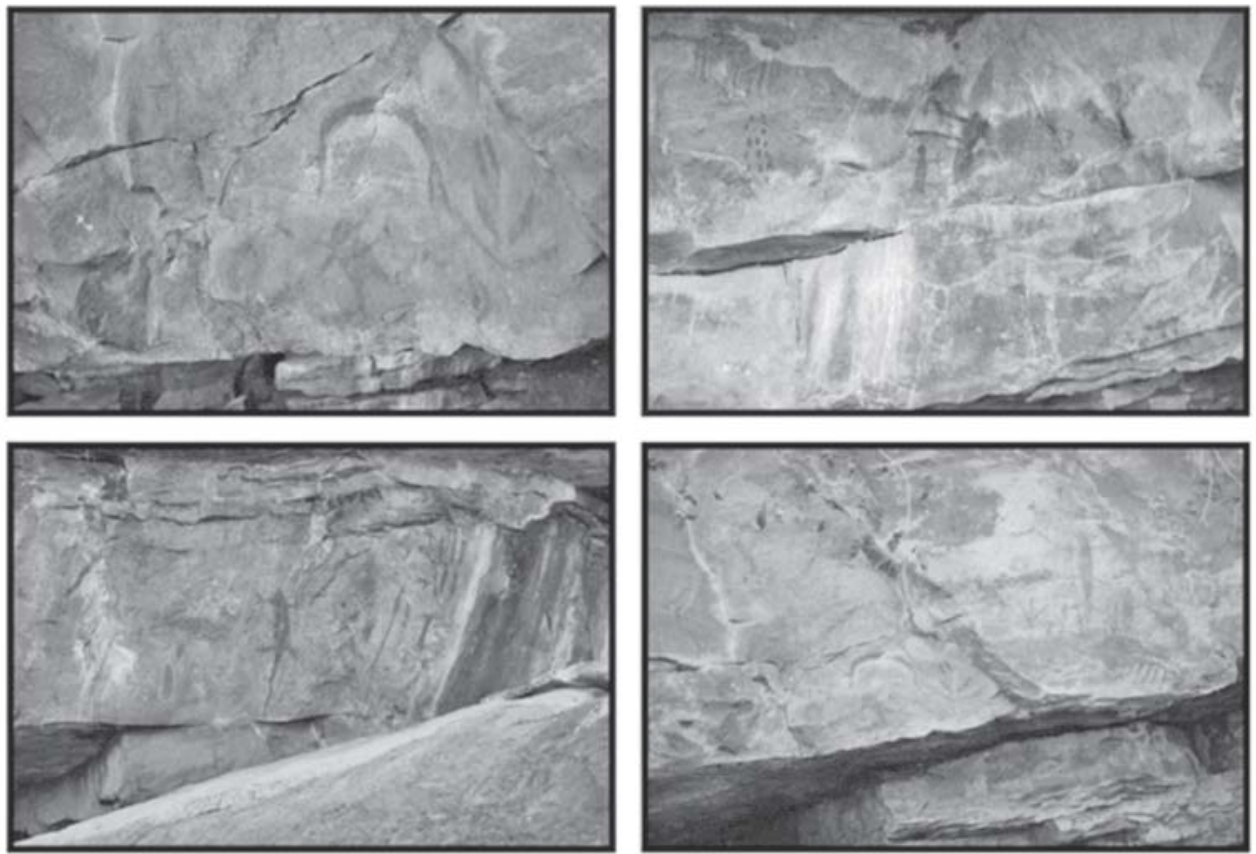

Gruta do Teú
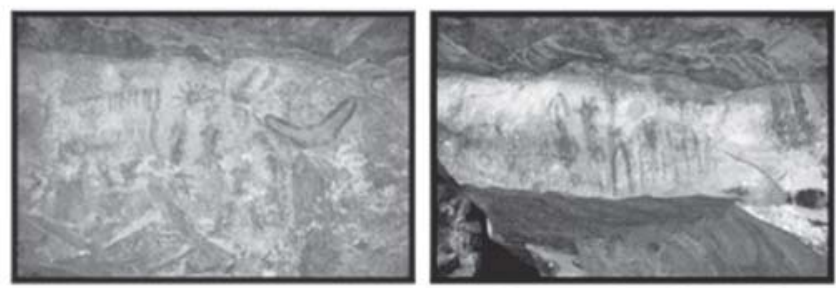

Gruta do Camé

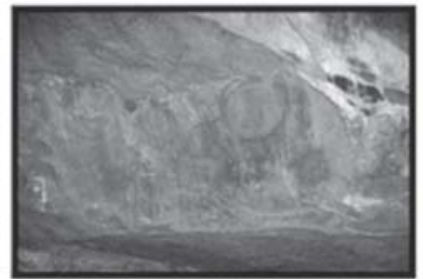

Fig. 2 
resolução de 30x30 metros, ou seja, cada pixel da imagem corresponde a um quadrante de $30 \mathrm{x}$ 30 metros. A partir da DEM foi confeccionado um mapa de declividade de terreno e outro que representa a direção da declividade. A estes dois mapas foram acrescidos um mapa de solo da área, um mapa hidrográfico e um mapa de vegetação. A partir desses dados foi produzido um mapa de custo. O segundo passo foi determinar o custo metabólico de deslocamento da área como um todo. Para isso utilizamos a fórmula como sugerida por Morin et al. (2005) por apresentar um resultado mais detalhado e adequado com a medição do gasto metabólico em volume de oxigênio consumido. $\mathrm{O}$ mapa de custo metabólico foi somado com o mapa de custo ambiental e o resultado final foi um mapa que nos mostra as áreas de menor custo geral de deslocamento, levando em conta fatores ambientais e fisiológicos. A partir deste mapa foram calculadas as rotas de direcionalidade inerente dentro da área de estudo. As rotas foram calculadas tomando por base a direção do movimento, assim foram produzidas rotas na direção S/N, N/S, W/E e E/W. O resultado é uma rede de permeabilidade do espaço ou mapa de trânsito teórico. Tais mapas oferecem uma amostra das rotas que podem ser potencialmente utilizadas com um mínimo esforço em circunstâncias concretas (Criado Boado 1999). Estas rotas representam a Direcionalidade Inerente do Meio.

Observando os mapas veremos que as áreas mais claras indicam locais onde o custo de deslocamento é menor e as áreas mais escuras áreas onde o consumo de oxigênio é maior, ou seja onde o custo de deslocamento é maior. Como podemos ver nos Mapas 3a a 3d, as rotas de direcionalidade inerente se localizam sobretudo no fundo dos vales e ao longo da calha de drenagem principal. $\mathrm{Na}$ área mais central do mapa podemos observar também uma série de caminhos que cruzam os topos de montanha, e as regiões mais planas do carste. É interessante notar que os caminhos na direção N/S e S/N, cruzam os sítios apenas na porção mais a oeste, ficando longe da área de maior concentração localizada na porção mais central do mapa. Como podemos observar, os três sítios a oeste são na realidade sítios a céu aberto e não sítios de abrigo. No caso dos caminhos posicionados nas direções $\mathrm{E} / \mathrm{W}$ e W/E, somente as rotas na direção $\mathrm{E} / \mathrm{W}$ cruzam a área dos sítios.

O passo seguinte foi confeccionar o mapa de Direcionalidade Cultural. Se pensarmos que na realidade percepção não é apenas condicionada pelo movimento, mas também pela observação, foi criado um mapa que representa a visibilidade a partir dos sítios arqueológicos. $\mathrm{O}$ modelamento de superfícies de visibilidade tem se destacado na arqueologia, principalmente porque percepção em arqueologia tem sido um fenômeno normalmente associado à visão e não ao movimento. Análises de Visibilidade identificam as áreas dentro de um determinado espaço que podem, ou não, ser vistas a partir de um ponto de observação. É possível determinar também de que localidades o ponto inicial pode ser avistado. Análises deste tipo são úteis principalmente quando se pretende determinar o campo de visão a partir de um dado ponto e se calcular as distâncias envolvidas. Em arqueologia, análises de visibilidade têm sido utilizadas na determinação do campo visual dos indivíduos a partir do assentamento e como se processava a interação entre as chamadas áreas visíveis e não visíveis da paisagem.

Mas basear percepção somente no aspecto visual nos parece uma simplificação inadequada já que o processo de percepção e experimentação da paisagem não deve se basear exclusivamente em conceitos visuais, pois existe o risco de cair no mesmo reducionismo incorporado às análises anteriores. Como Gillings e Godrick (1996) sugerem, uma noção mais abrangente de percepção deve ser baseada não somente no aspecto visual, mas na recepção multi-sensorial de informações tendo como principio a mobilidade e o deslocamento dos indivíduos. Movimento e ação estabelecem as bases da percepção.

Desta maneira, o mapa de Direcionalidade Cultural levou em conta não somente a superfície de gasto metabólico como também a superfície de visibilidade. Em seguida, foram calculadas as rotas de deslocamento a partir da localização dos sítios arqueológicos, principalmente dos abrigos com pintura. Como podemos ver nos Mapas 4a a 4d, as principais rotas de desloca- 
Uma Fisiologia da Paisagem II: Percepção e Movimento.

Revista do Museu de Arqueologia e Etnologia, São Paulo, 18: 3-18, 2008.
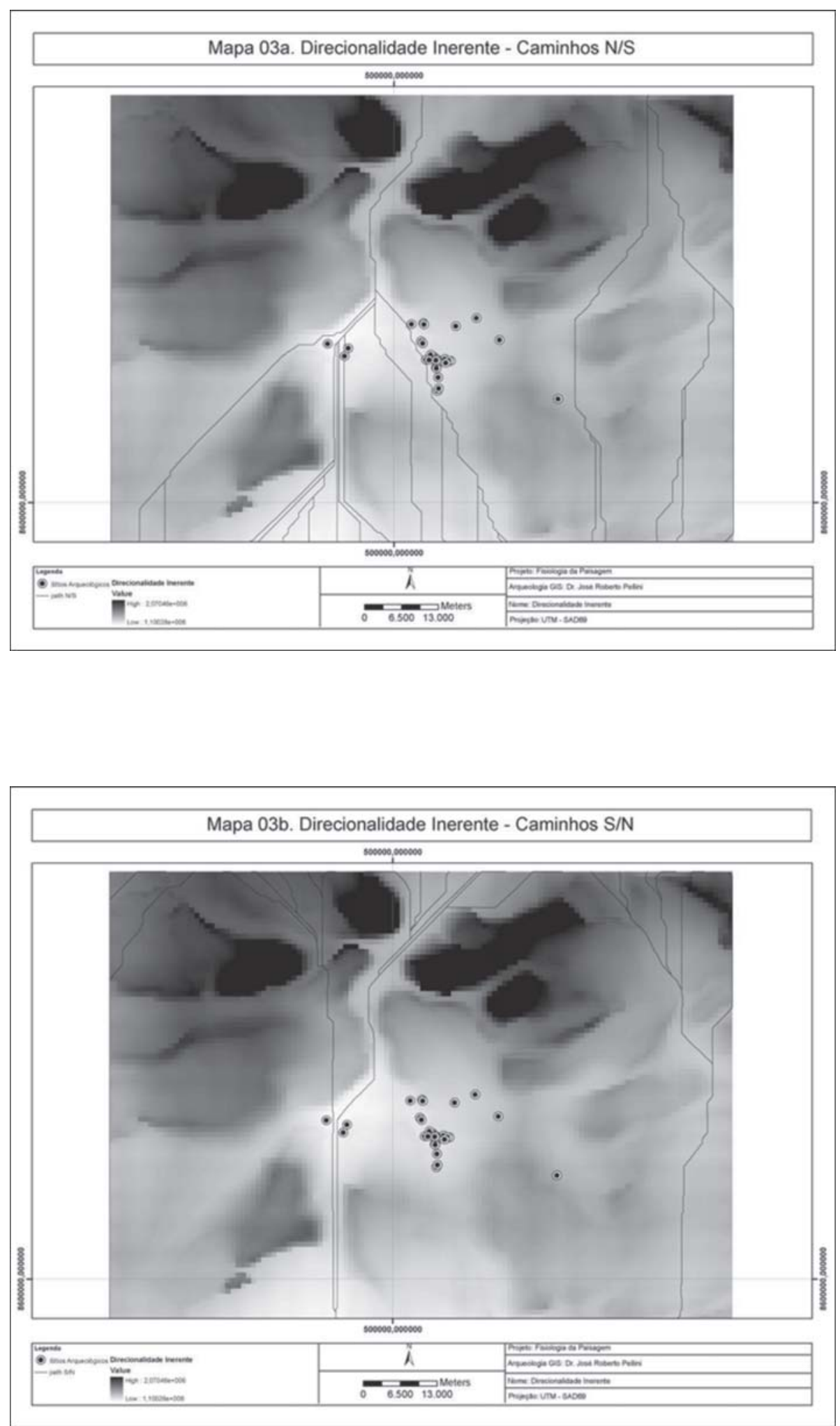

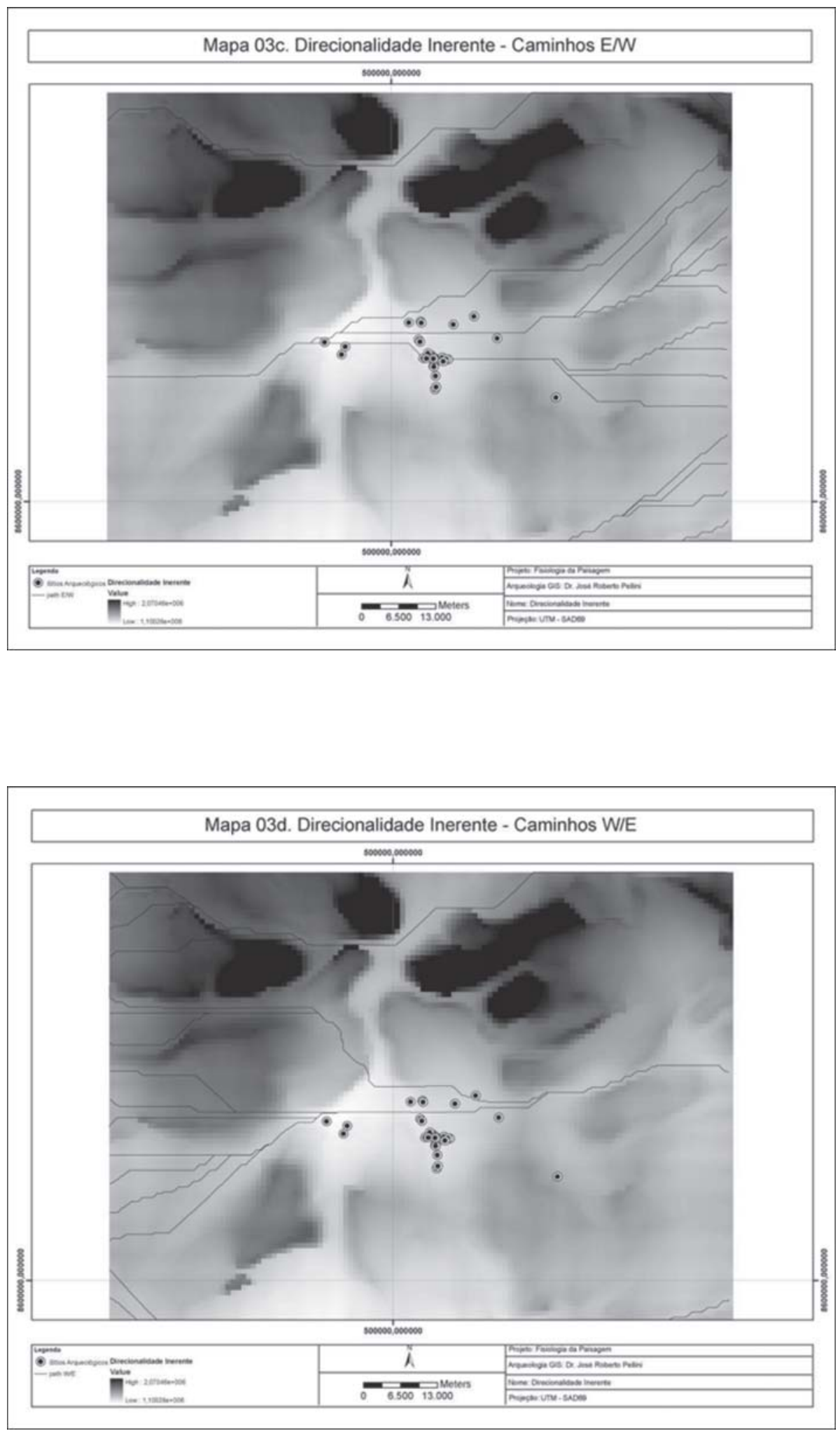
Uma Fisiologia da Paisagem II: Percepção e Movimento.

Revista do Museu de Arqueologia e Etnologia, São Paulo, 18: 3-18, 2008.
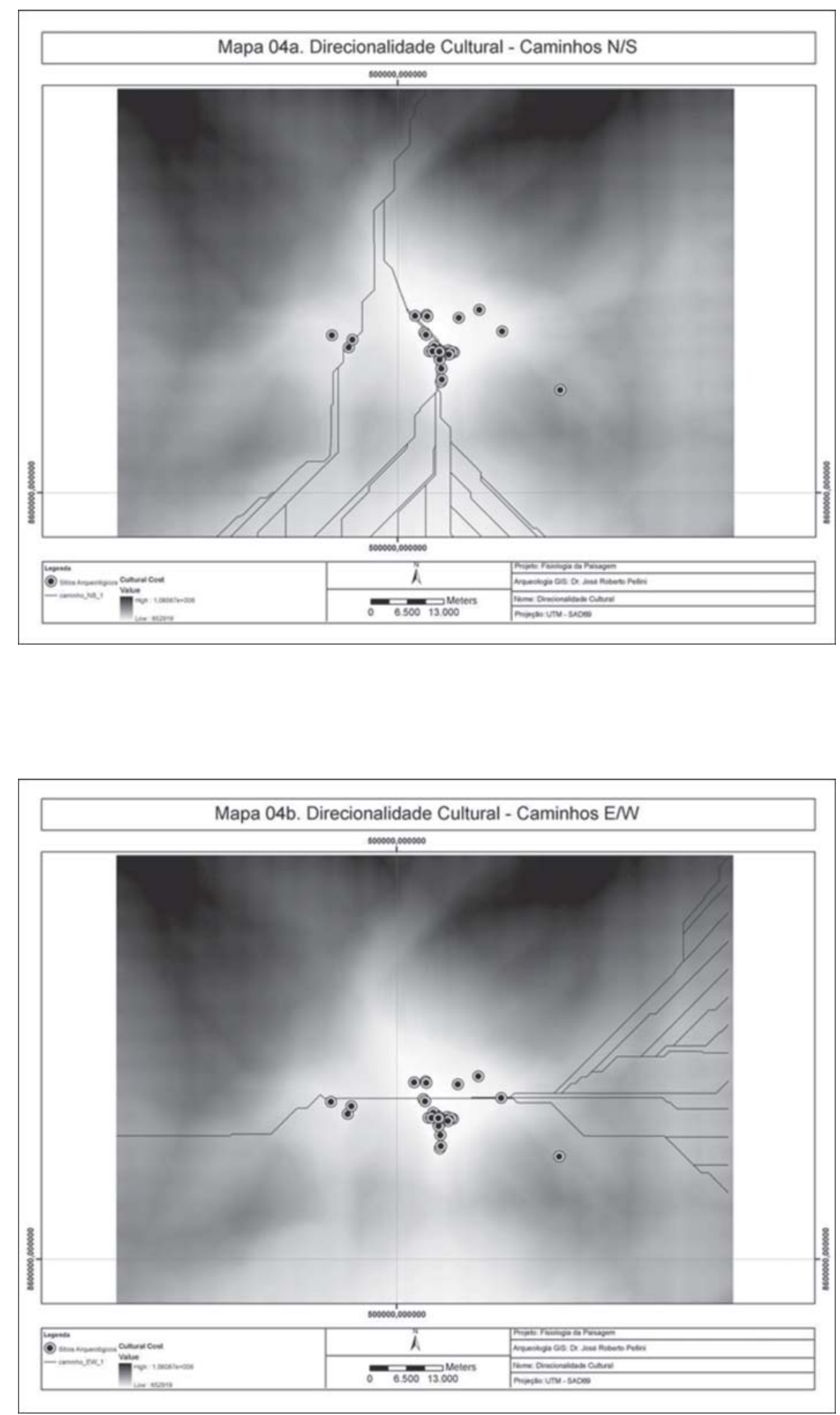

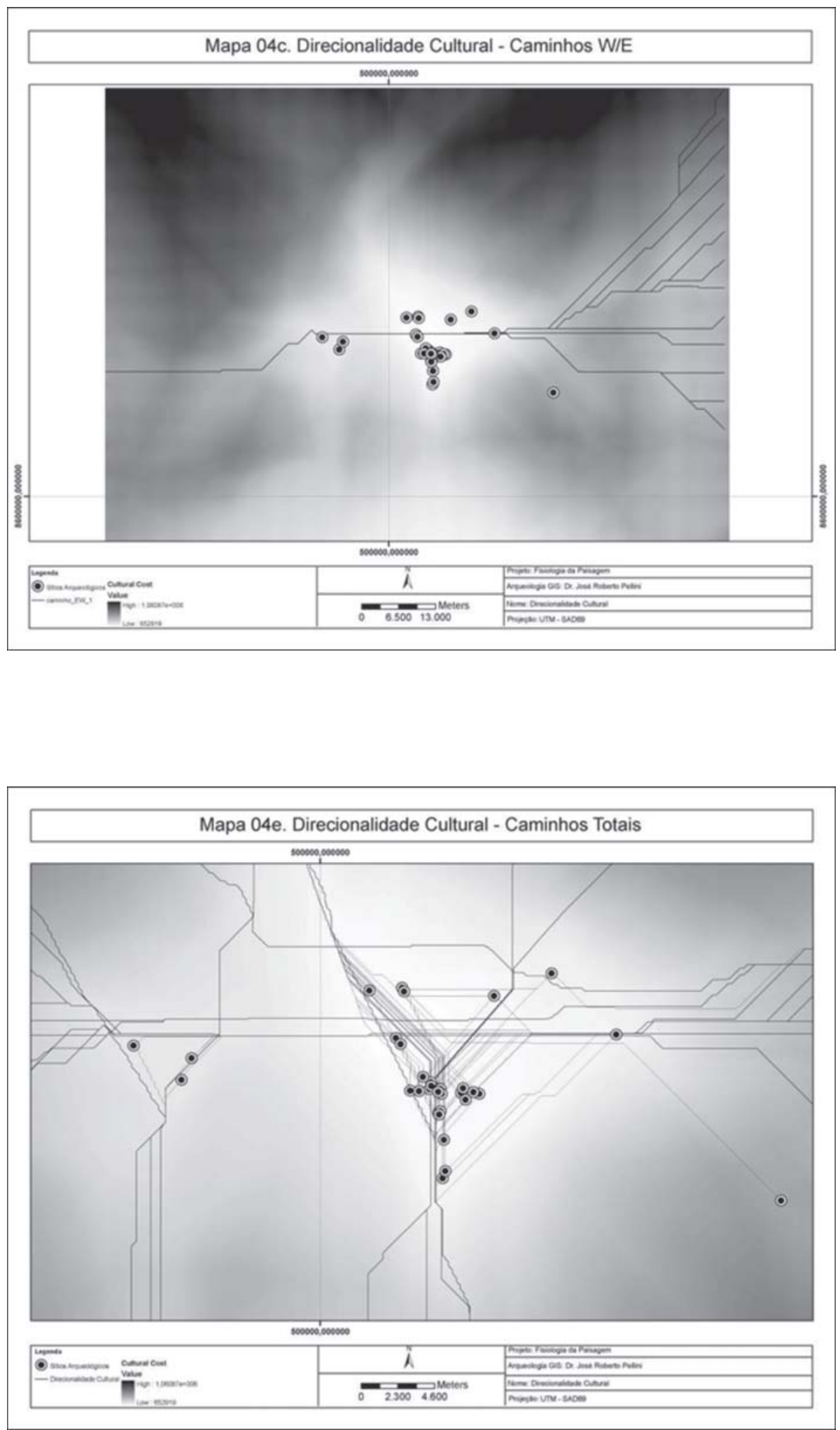
mento passam por entre o conjunto de abrigos que se localiza na porção mais central da área de estudo. Neste conjunto se localizam os sítios abrigo com pintura. É de se destacar a rota N/S, pois é a que dá maior acesso aos sítios arqueológicos de maneira geral, já que as demais rotas passam apenas no centro do agrupamento. Do centro do agrupamento saem rotas que interligam todos os sítios como fica claro no Mapa 4e que mostra com detalhe a relação dos sítios com as rotas calculadas.

Comparando ambas as rotas, a de direcionalidade inerente e a de direcionalidade cultural, podemos perceber que há uma significativa diferença nos caminhos de deslocamento. No caso dos caminhos afetados pela direcionalidade inerente temos uma profusão de rotas principalmente nas áreas limites do mapa. Já no caso dos caminhos de direcionalidade cultural, embora ainda haja uma grande quantidade de caminhos nas áreas limites, a principal diferença se dá na área mais próxima aos sítios arqueológicos. Não somente as rotas se afunilam para se tornarem únicas como há uma maior interligação entre os sítios. Desta maneira podemos dizer que o fator de atratividade nas rotas culturais não estava condicionado pelo mínimo esforço, mas por diferentes fatores sócio-culturais que se sobrepunham aos atributos naturais do terreno. Como a principal atratividade se dá no núcleo de maior concentração de abrigos e estes apresentam uma grande quantidade de pinturas rupestres, é possivel pensar que tais abrigos funcionem como centro de atração social ou cerimonial. Tal atratividade seria forte $\mathrm{o}$ suficiente para que os chamados caminhos de menor custo ou as rotas de deslocamento naturais fossem modificadas para favorecer pelo menos sazonalmente $\mathrm{o}$ acesso aos abrigos.
Isto mostra como fatores de minimização de custo e energia nem sempre condicionam o movimento e que devemos ter cuidado ao utilizar as noções de racionalidade econômica. Fatores sociais, econômicos e religiosos são aspectos que não podem ser deixados de lado quando tratamos dos padrões de mobilidade e das redes de permeabilidade espacial.

Embora o trabalho ainda não tenha se encerrado já que novos refinamentos necessitam ser aplicados aos modelos de análise, a tentativa foi desenvolver novos modelos que possam superar os modelos espaciais funcionalistas desenvolvidos pela arqueologia processual. Assim como Llobera (2000) propõe a análise da sociologia do movimento tentando identificar não caminhos concretos, mas sim tendências gerais de movimento focando principalmente nos fatores que condicionam sua criação, uso e evolução, tentamos verificar em que medida aspectos naturais, fisiológicos e culturais determinam e alteram a rede de permeabilidade especial. As análises de movimento e deslocamento não devem se restringir a aspectos naturais do terreno, pois devem incluir a possibilidade de que os elementos culturais atuem como foco de atração ou repulsão da mobilidade e das rotas de movimento.

Ao mesmo tempo, como pudemos ver acima, análises de movimento e visibilidade e, portanto, de percepção da paisagem oferecem um campo fértil de pesquisa e interpretação que nos permitem um olhar diferente sobre as antigas paisagens e o desenvolvimento de novos modelos de análise em arqueologia que ultrapassam os modelos deterministas que vem sendo aplicados ao GIS. 
PELLINI, J.R. A Physiology of Landscape II: Perception and Movement. Revista do Museu de Arqueologia e Etnologia, São Paulo, 18: 3-18, 2008.

\begin{abstract}
Thinking that perception is a function of the movement we can say that the way in which individuals perceive their environment depends on how they move. If individuals change their perception of the environment through the movement, the spatial dimension in archaeology can be conceptualized from the profiles of mobility of individuals. Today, the methods of analysis in GIS enable the reconstruction of the patterns of human mobility using the latest research on physiology of the movement and the so-called analysis of visibility. This enables the development of approaches that emphasize cognitive aspects related to the perception and movement and thus the creation of the GIS tools that go beyond the notion of Cartesian space.
\end{abstract}

Keywords: GIS - Perception - Movement - Landscape.

\title{
Referências bibliográficas
}

ABBOT, B.; BIGLAND, B.; RITCHIE, J.

1952 The Physiological Cost of Negative Work. Journal of Physiology, 117: 380-390.

BENDER, B.

2001 Introduction. In: Bender, B.; Winer, M. (Eds.) Contested Landscapes. Movement, Exile and Place. Oxford, Berg: 1-20.

BOURDIEU, P.

1977 Outline of a Theory of Practice. Cambridge: Cambridge University Press.

CRIADO BOADO, F.

1999 Del Terreno al Espacio: Planteamientos y Perspectivas para la Arqueologia Paisaje. Capa 6. Compostela, Universidad de Santiago de Compostela.

GIBSON, J.

1979 The Ecological Approach to Visual Perception. Hillsdale, New Jersey: Lawrence Erlbaum Association.

GILLINGS, M.; GOODRICK, G.

1996 Sensuous and Reflexive GIS: Exploring Visualization and VRML. Internet archaeology 1. (HTTP://intach.ac.uk/ journal/issue1/gillings_toc.html)

HÄGERSTRAND, T.

1962 Geographical Measurements of Migration. In: Sutter, J. (Ed.) Les Desplacements Humains. Aspects Methodologiques de Leur Mesure. Entretiens de Monaco en Sciences Humaines, 24-29 Mai, 1962: 31-62.
INGOLD, T.

1986 Territoriality and Tenure: The Appropriation of Space in Hunting and Gathering Societies. In: Ingold, T. (Ed.) The Appropriation of Nature. Manchester, Manchester University Press: 130-164.

2000 The Perception of the Environment. Essays on Livelihood, Dwelling and Skill. London: Routledge.

LEGAT, A.

2008 Walking Stories: Leaving Footprints. Ingold, T.; Vergunst, J.L. (Eds.) Ways of Walking. Ethnography and Pratice on Foot. Anthropological Studies of Creativity and Perception. Ashgate, Hampishire, England: 35-50.

LEGG, S.

1985 Comparasion of Different Methods of Load Carriage. Ergonomics, 28: 197-212.

LEGG, S.; MAHANTY, A.

2004 Comparison of Five Modes of Carryng a Load Close to the Trunk. Ergonomics, 28: 1653-1660.

LLOBERA, M.

2000 Understand Movement: A Pilot Model Towards the Sociology of Movement. In: Lock, G. (Ed.) Beyond the Map: Archaeology and Spatial Technologies. Amsterdam, IOS Press: 65-84.

MACKIE, Q.

2001 Settlement Archaeology in a Fjordland 
Archipelago. Network analysis, Social Practice and a Built Environment of Western

Vancouver Island, British Columbia, Canada, since 2000 BP. BAR Series 926. Oxford: Archaeopress.

MILLER, H.J.; WENTZ, E.A.

2003 Representation and Spatial Analysis in Geographic Information Systems. Annals of the Association of American Geographers, 93: 574-594.

MINETTI, A.; ARDIGÓ, L.; SAIBENE, F.

1994 Mechanical Determinants of the Minimum Energy Cost of Gradient Runnings in Humans. Journal of Experimental Biology, 195: 211-225.

MINETTI, A.; MOIA, C.; ROI, G.; SUSTA, D.; FERRETTI, G.

2002 Energy Cost of Walking and Running at Extreme Uphill and Downhill Slopes. Journal of Applied Pysiology, 93: 1039-1046.

MOLES, K.

2008 A Walk in Thirdspace: Place, Methods and Walking. Sociological Research Online, $13(4): 1-10$.

MORIN, E.Ç; STEVENSON, J.Ç; REID, S.; BRYANT, J.

2005 Development of a Dynamic Biomechanical Model for Load Carriage: Phase V: Development of the Dynamic Biomechanical Model by Means of the Portable Measurement System. Department of National Defense. Defense Research and Development Canada, Toronto.

MORRILL, R.; PITTS, F.

1967 Marriage, Migration and the Mean Information Field: A Study in Uniqueness and Generality. Annals of the Americam Association of Geographers, 57 (2): 401-422.
PANDOLF, K.; GIVONI, B.; GOLDMAN, R.

1977 Predicting Energy Expediture with Loadas While Standing or Walking Very Slowly. Journal of Applied Physiology, 43: 577-581.

PELLINI, J.R.; TELLES, M.

2008 Projeto de Resgate Arqueológico, Histórico e Cultural da Área Direta e Indiretamente Afetada pala Implantação da Rodovia BR 135. Griphus Consultoria. Goiânia, Goiás.

PONTZER, $\mathrm{H}$.

2007 Predicting the Energy Cost of Terrestrial Locomotion. A Teste of the LIMB Model in Humans and Quadrupeds. Journal of Experimental Biology, 210 (3): 484-494.

SANTEE, W; BLANCHARD, L; SPECKMAN, K; GONZALEZ, J; WALLACE, R.

2003 Load Carriage Model Development and Testing with Field Data. US Army Research Institut of Environmental Medicine. Technical Note. Kansas City.

TILLEY, C.

1994 A Phenomenology of Landscape. Places Paths and Monuments. Oxford: Berg.

TOBLER, W.

1993 Non-isotropic Geographic Modeling. Three Presentation on Geographical Analysis and Modeling. National Center for Geographic Information and Analysis. Techical Report, 93 (1).

TUCK PO, L.

2008 Before a Step Too Far: Walking with Batek Hunter Gatherers in the Forests of Pahang, Malasya. In: Ingold, T.; Vergunst, J.L. (Eds.) Ways of Walking. Ethnography and Pratice on Foot. Anthropological Studies of Creativity and Perception. Ashgate, Hampishire, England: 21-34. 